\title{
Multivisceral lgG4-related disease presenting as recurrent massive gastrointestinal bleeding: a case report and literature review
}

\author{
Xuexue Deng, Ronghua Fang ${ }^{*}$ D, Jianshu Zhang and Rongqiong Li
}

\begin{abstract}
Background: IgG4-related disease (IgG4-RD) is a newly recognized autoimmune systemic disorder characterized by elevated levels of serum IgG4 and abundant infiltration of IgG4-positive plasmacytes in the affected organs. The liver, biliary system and pancreas are the most commonly affected organs. However, involvement of the digestive tract is very rare. To date, only a few cases of isolated gastric IgG4-RD have been reported.

Case presentation: We present a case of IgG4-RD of the liver, gallbladder, pancreas and duodenum, which was clinically misinterpreted and thereafter over-treated. A 52-year-old male presented with obstructive jaundice for 3 years, melena for 5 months and hematemesis for 10 days. Three years prior, the patient had undergone biopsies of pancreatic lesions, liver lesions, cholecystectomy and choledochojejunostomy. Histopathology showed chronic inflammatory changes. Endoscopy at admission revealed a duodenal ulcer with active bleeding. Despite medical management, the patient presented with repeated gastrointestinal bleeding. Upon evaluation, serum IgG4 levels were found to be elevated. Histopathology of the duodenal ulcer biopsy and repeated examination of the gallbladder and pancreatic and liver biopsies confirmed IgG4 positive plasma cell infiltration. A definitive diagnosis of IgG4-RD was made and steroid administration was initiated. At last follow up, 11 months to-the-day after initiating steroid treatment, the patient was asymptomatic.
\end{abstract}

Conclusions: Notably, IgG4-RD of multiple digestive organs is still very rare. As a systemic disease, it is characterized by the infiltration of IgG4-bearing plasma cells and raised lgG4 levels. Histopathology findings remain the diagnostic gold standard for this disorder.

Keywords: IgG4-related disease, Plasma cells, Liver, Gallbladder, Duodenal, Pancreatic, Gastrointestinal bleeding

\section{Background}

IgG4-related disease (IgG4-RD) is a relatively recently recognized chronic multi-organ autoimmune disease of unknown origin with a tendency to develop lesions at multiple sites throughout the body [1]. It is known to affect various organs including the bile duct, pancreas, gallbladder, liver, lungs and salivary glands. It is characterized by elevated IgG4 levels in the serum and the affected tissues, lymphoproliferative infiltration and sclerotic fibrosis $[2,3]$. Other typical features are occlusive phlebitis and

\footnotetext{
* Correspondence: fangronghua@126.com

Department of General Medicine, West China Hospital, Sichuan University, No. 37 Guoxuexiang, Wuhou District, Chengdu City, Sichuan Province, China
}

rising IgG4-plasma cells levels $[4,5]$. Clinically, it mimics malignancy in some patients, especially those with liver and pancreatic lesions and is often thought to necessitate surgical resection, sometimes leading to over-treatment $[6,7]$. To date, only a few isolated cases of digestive IgG4-RD have been reported.

\section{Case presentation}

A 52-year-old male from southwest China presented with obstructive jaundice for 3 years, melena for 5 months and hematemesis for 10 days. The patient had been diagnosed with type 2 diabetes mellitus for 4 years, which was treated with metformin. The patient was a

(c) The Author(s). 2018 Open Access This article is distributed under the terms of the Creative Commons Attribution 4.0 International License (http://creativecommons.org/licenses/by/4.0/), which permits unrestricted use, distribution, and 
Table 1 Comparison of lgG4 parameters and routine blood and markers for liver function before and after treatment

\begin{tabular}{|c|c|c|c|c|c|c|c|}
\hline Parameter & Admission day & Confirmed day & $\begin{array}{l}\text { After treatment } \\
8 \text { days }\end{array}$ & $\begin{array}{l}\text { Follow up } \\
1 \text { month }\end{array}$ & $\begin{array}{l}\text { Follow up } \\
3 \text { months }\end{array}$ & $\begin{array}{l}\text { Follow up } \\
6 \text { months }\end{array}$ & $\begin{array}{l}\text { Follow up } \\
9 \text { months }\end{array}$ \\
\hline $\operatorname{lgG4}(\mathrm{g} / \mathrm{L})$ & - & 29.20 & 18.40 & 9.86 & 4.74 & 1.87 & 1.70 \\
\hline $\lg G(g / L)$ & - & 24.5 & 19.4 & - & - & 8.80 & - \\
\hline CRP (mg/ L) & 5.46 & - & - & - & 2.62 & 1.32 & - \\
\hline TBIL $(\mu \mathrm{mol} / \mathrm{L})$ & 12.1 & 11.7 & 56.4 & 23.4 & 14.1 & 7.7 & - \\
\hline DBIL (umol/L) & 6.5 & 5.4 & 44.5 & 16.4 & 6.1 & 3.1 & - \\
\hline ALT (IU/L) & 41 & 13 & 315 & 134 & 44 & 29 & - \\
\hline AST (IU/L) & 40 & 24 & 167 & 57 & 28 & 26 & - \\
\hline ALB (g/L) & 30 & 36.4 & 34.8 & 37.1 & 35.2 & 42.1 & - \\
\hline $\operatorname{PLT}\left({ }^{*} 10^{9} / \mathrm{L}\right)$ & 80 & 95 & 88 & 106 & 81 & 110 & 140 \\
\hline $\mathrm{HGB}(\mathrm{g} / \mathrm{L})$ & 75 & 61 & 98 & 71 & 122 & 114 & 123 \\
\hline
\end{tabular}

IgG4 Immunoglobulin G4 subtype, IgG Immunoglobulin G, CRP C-reactive protein, TBIL total bilirubin, DBIL direct bilirubin, ALT alanine aminotransferase, AST aspartate aminotransferase, $A L B$ albumin, PLT platelet, $H G B$ hemoglobin

chronic tobacco (10 cigarettes per day) and alcohol consumer over the previous 20 years but had quit smoking and drinking for the past 3 years. Three years prior to admission to our hospital, he developed obstructive jaundice, which was investigated with abdominal computed tomography (CT) and revealed multiple hypodense lesions in the liver and pancreatic head mass. He underwent cholecystectomy, choledochojejunostomy and biopsies from the liver and pancreatic lesions. Histopathology revealed chronic cholecystitis with lymphocytic, plasmacytic and eosinophilic infiltration of the gallbladder, liver and pancreatic parenchyma.
The physical examination was unremarkable (height $165 \mathrm{~cm}$, weight $-50 \mathrm{~kg}$ ). Laboratory tests revealed hemoglobin levels of $75 \mathrm{~g} / \mathrm{L}$, a platelet count of $80 \times 10^{9} / \mathrm{L}$, $30.0 \mathrm{~g} / \mathrm{L}$ albumin levels, and serum potassium levels of $3.20 \mathrm{mmol} / \mathrm{L}$ (Table 1). The fecal occult blood was positive.

On the day of admission, the patient developed repeated painless hematemesis of about $2100 \mathrm{~mL}$, which was associated with hypotension and tachycardia. He was resuscitated with intravenous, blood and norepinephrine infusions. Laboratory tests revealed hemoglobin levels of $47.5 \mathrm{~g} / \mathrm{L}$, platelet count levels of $38 \times 10^{9} \mathrm{~L}$ and

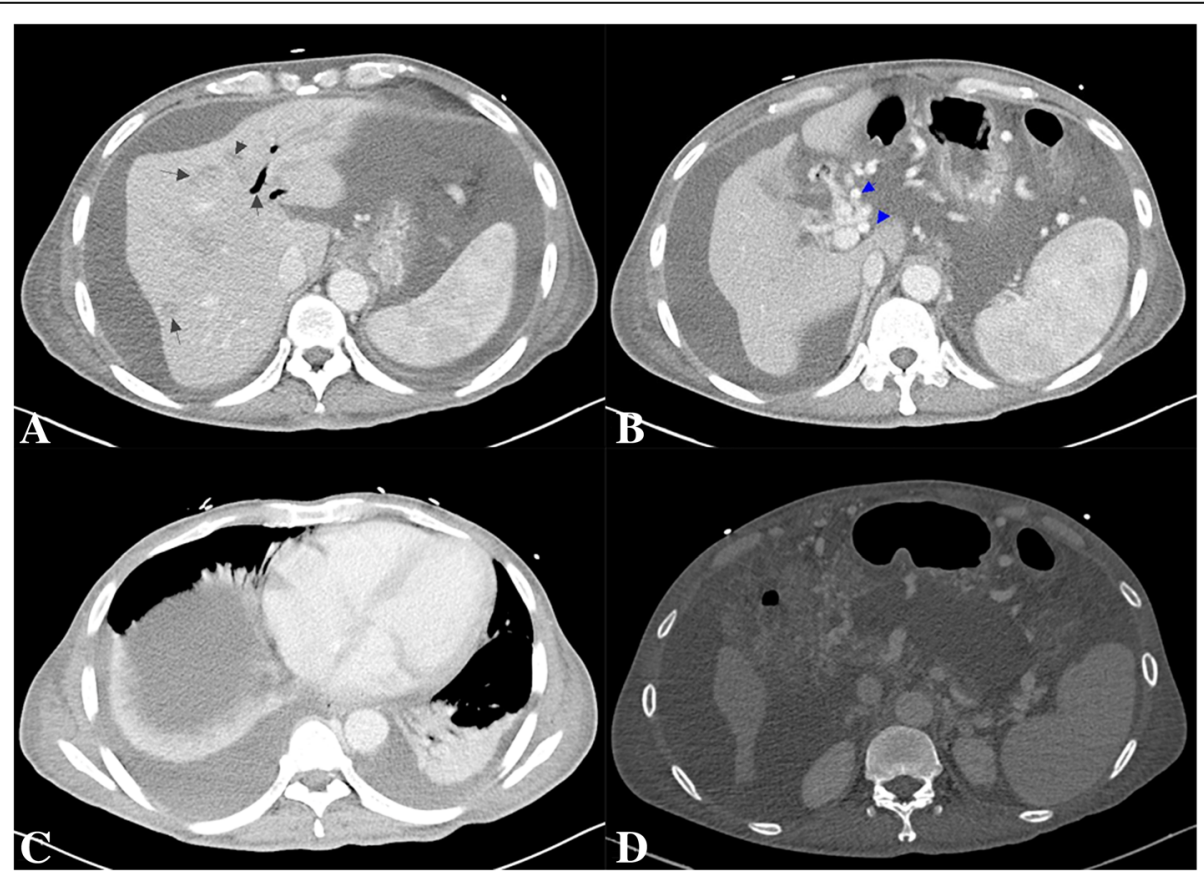

Fig. 1 Abdominal computed tomography. Images of the liver showed numerous hypodense nodules with mild contrast enhancement, ascites and intrahepatic bile duct dilatation (a). There were multiple enlarged lymph nodes in the hepatoduodenal ligament around the abdominal aorta (b). Mesenteric lymph nodes with bilateral pleural effusion (c). Multiple nodular dense shadows with dilatation of the main pancreatic duct in the tail region $(\mathbf{d})$ 


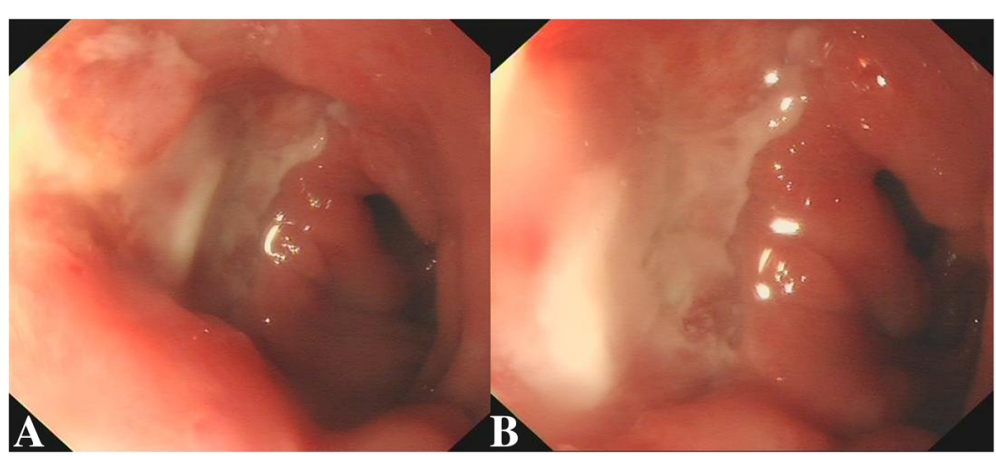

Fig. 2 Gastroscopy findings. An ulcer $(1.5 \mathrm{~cm} \times 1.2 \mathrm{~cm})$ in the anterior wall of the duodenal bulb and a lot of white mosses in the fundus of stomach (a, b)

serum albumin $25.8 \mathrm{~g} / \mathrm{L}$. The day after admission, the patient again developed hematemesis of about $500 \mathrm{~mL}$, for which he was treated with somatostatin, terlipressin and pantoprazole infusion. Sengstaken-Blakemore tube were placed to hemostasis by compression and tube feeding hemostatic medicine. After 4 days of medical intervention, the patient's gastrointestinal bleeding gradually stopped. Esophagogastroduodenoscopy revealed a duodenal ulcer (A1 stage) with active bleeding, gastric mucosal erosions and esophageal varices (mild). Contrast enhanced computed tomography of the abdomen with three-dimensional reconstruction exposed multiple nodular dense shadows with dilatation of the main pancreatic duct in the tail region (Fig. 1d). Images of the liver showed numerous hypodense nodules with mild contrast enhancement, ascites and intrahepatic bile duct dilatation (Fig. 1a). Additionally, there were multiple enlarged lymph nodes in the hepatoduodenal ligament around the abdominal aorta (Fig. 1b) and mesenteric lymph nodes with bilateral pleural effusion (Fig. 1c). In view of the liver disease with portal hypertension and esophagogastric varices, a transjugular intrahepatic

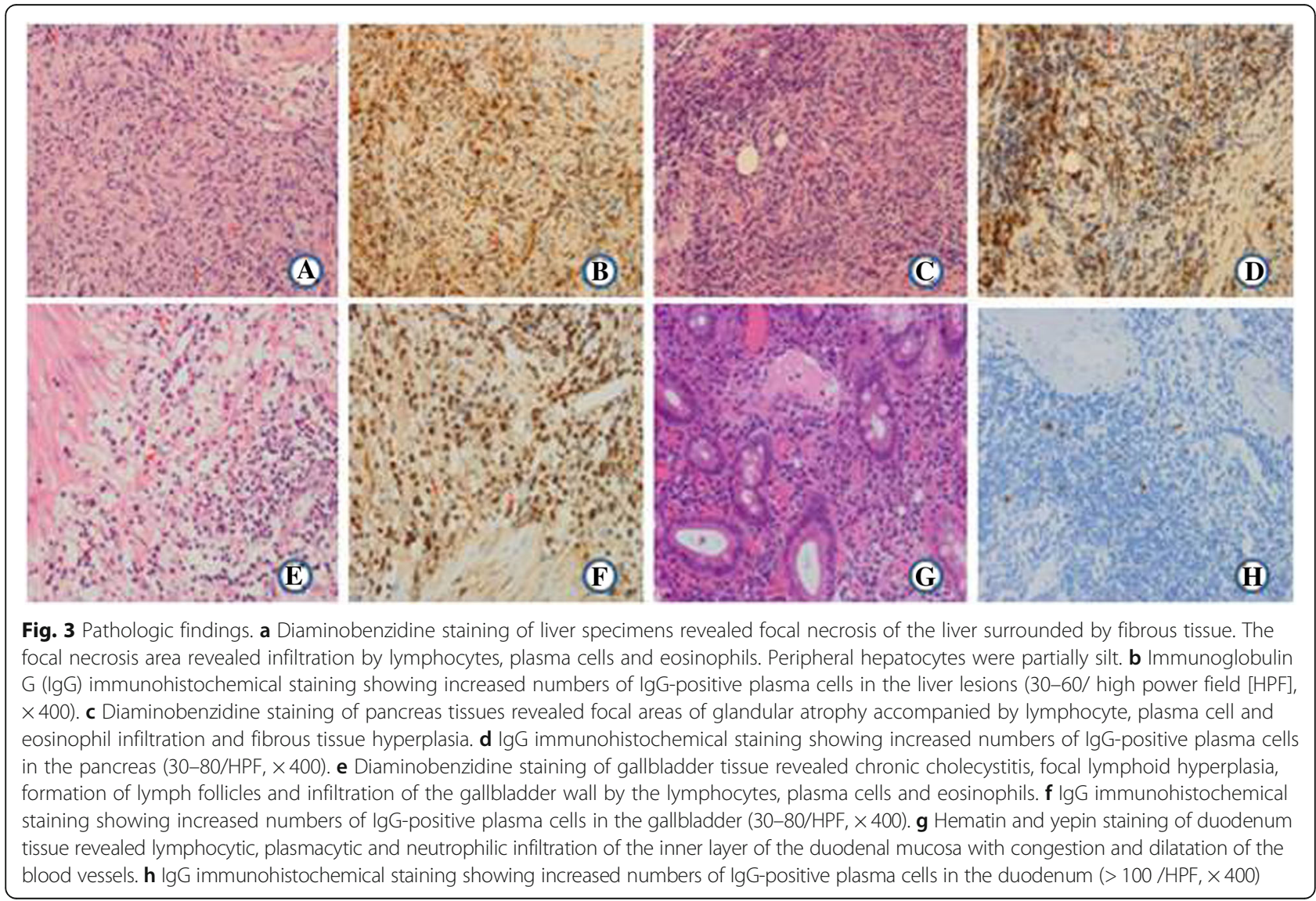




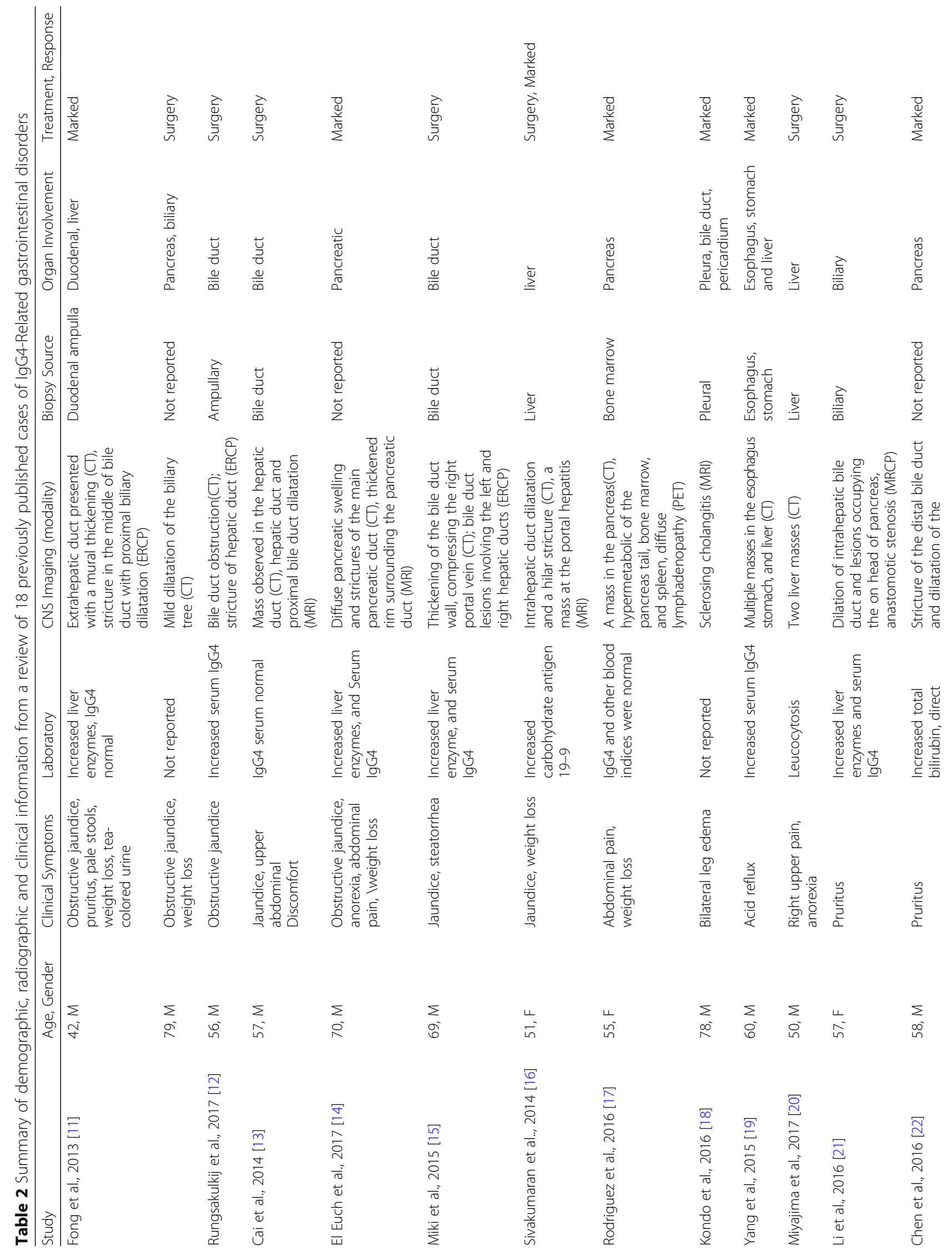


Deng et al. BMC Gastroenterology (2018) 18:136

Page 5 of 7

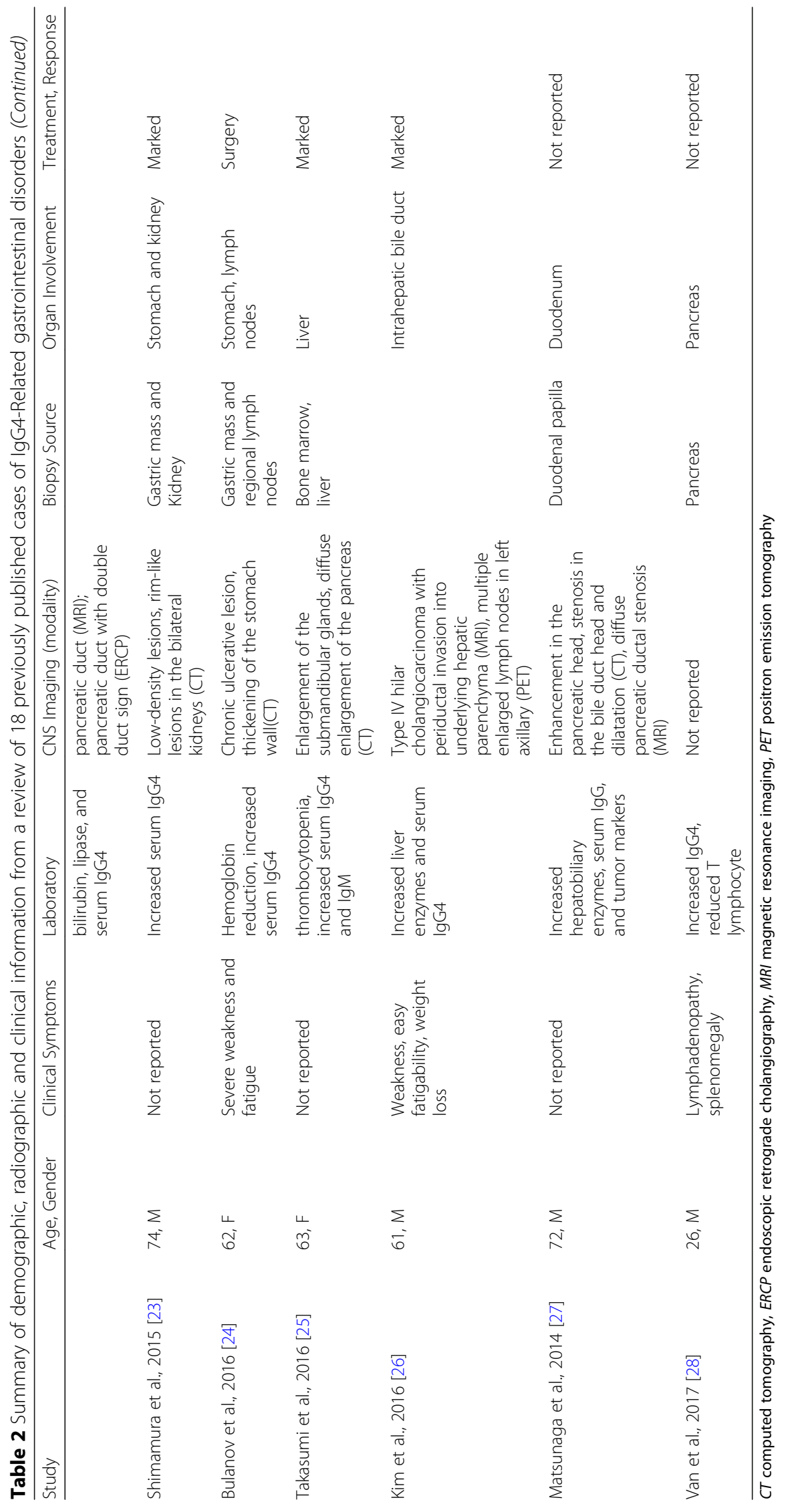


portosystemic shunt (TIPS) procedure was performed under local anesthesia and regional portal hypertension, splenic vein and portal vein obstruction were detected. Subsequently, the patient re-bleed both 14 days and 20 days after admission, about $600 \mathrm{~mL}$ and $400 \mathrm{~mL}$, respectively. The patient was treated with somatostatin, pantoprazole infusion and blood transfusion. Gastroscopy identified an ulcer $(1.5 \mathrm{~cm} \times 1.2 \mathrm{~cm})$ in the anterior wall of the duodenal bulb that was not actively bleeding (Fig. 2a, Fig. 2b). Pathological examination of the ulcer biopsy verified a moderate degree of chronic mucosal inflammation.

When investigating the recurrent bleeds, serum $\operatorname{IgG4}(0.035-1.500 \mathrm{~g} / \mathrm{L})$ and $\operatorname{IgG}(8 \sim 15.5 \mathrm{~g} / \mathrm{L})$ were found to be $29.200 \mathrm{~g} / \mathrm{L}$ and $24.50 \mathrm{~g} / \mathrm{L}$ respectively and IgG4 disease was suspected (Table 1). The patient's prior surgical pathology specimens were retrieved for IgG4 immunohistochemical examination. Immunohistochemical staining revealed increased IgG-positive and IgG4-positive plasma cells in the liver lesions (IgG4 positive cells 30-60/ high power field (HPF); Fig. 3b), pancreas (IgG4 positive cells 30-80/HPF; Fig. 3d) and gallbladder (IgG4 positive cells 30-80/HPF; Fig. 3f). Staining of the duodenum ulcer biopsy also showed more than 100 IgG4-positive cells per HPF (Fig. 3h). Based on these findings, a definitive diagnosis of IgG4-RD was made.

The patient was administered $40 \mathrm{mg} / \mathrm{d}$ prednisone intravenously for 7 days without any side effects followed by oral prednisolone $40 \mathrm{mg} / \mathrm{d}$ for 1 week. Laboratory tests indicated a reduction in the serum IgG4 levels 8 days after initiating prednisone but the liver function continued to be impaired (Table 1). As the patient improved symptomatically, he was discharged on a regime of prednisolone and mycophenolate mofetil.

Since discharge until the time of writing, during the 11 monthly visits since starting steroid therapy, laboratory tests indicate significant improvements in the patient's serum IgG4 subtype, liver function and hemogram tests (Table 1).

\section{Discussion and conclusions}

IgG4-RD was first described in patients with sclerosing cholangitis, known as autoimmune pancreatitis (AIP) type I. Subsequently, IgG4-RD was found to affect other organs [6]. At present IgG4-RD is believed to be a systemic disease and diagnostic criteria for IgG4-RD $[8,9]$ include: (1) single or multiple organs with diffuse or localized swelling, masses, nodules and/or hypertrophic lesions; (2) elevated serum IgG4 levels ( $\geq 135 \mathrm{mg} / \mathrm{dL}$ ); and (3) histopathologic features that include marked lymphocytic and plasma cell infiltration and fibrosis, with IgG4-positive plasma cell infiltration (IgG4/IgG positive cell ratio of $40 \%$ and IgG4-positive plasma cells exceeding 10/HPF). Diagnosis of IgG4-related is confirmed when all of the following are fulfilled: (1), (2) and (3). Diagnosis is likely if criteria $(1)+(3)$ are fulfilled, possible if $(1)+(2)$ are fulfilled and unlikely if only (1) presents.

Notably, the biliary tract and pancreas are the organs most frequently affected by IgG4-RD [10]. Few studies have reported isolated cases of IgG4-RD in other digestive organs, such as in the esophagus, stomach, liver or duodenum [7].

A recent review of IgG4-RD cases reporting on gastrointestinal lesions showed that most patients present with multiple organ disease involvement, including the stomach, duodenum, liver, bile duct, esophagus, jejunum, lymph nodes and pancreas (Table 2). Six patients presented with obstructive jaundice as the main clinical manifestation [11-16] and others presented with varied clinical symptoms [17-28]. Gastrointestinal bleeding was not reported in any of the studies. Our patient had recurrent gastrointestinal bleeding due to a duodenal ulcer. The ulcer failed to heal with standard treatment. Serum IgG4 was elevated in most of the cases reviewed, similar to our case, though was normal in three reports $[11,13,17]$ and was not reported in two cases $[11,18]$. The diagnosis of IgG4-RD was based on a combination of features that include clinical parameters, serology, immunohistochemistry, imaging and histopathology. In the present case, the diagnosis of IgG4-RD was initially missed in the histological analysis of the liver and pancreatic biopsy and gallbladder samples. When IgG4-RD was suspected based on the clinical and laboratory findings, IgG4 immunostaining was undertaken and the IgG4-RD diagnosis was confirmed. In our literature review, there were eight patients treated through surgery, 10 patients by corticosteroids, two were given corticosteroids after surgery, and in two patient studies, the treatment course was not reported (Table 2). Our patient responded partially to steroid therapy and required other immunosuppressive agents for a complete and successful response.

IgG4-RD can affect multiple gastrointestinal organs simultaneously or over different time periods. The clinical manifestations can mimic malignancy or other benign diseases such as portal hypertension, acid peptic disease. A high index of suspicion is required to make an accurate diagnosis and avoid unnecessary surgical interventions.

\section{Abbreviations \\ ALT: Alanine aminotransferase; AST: Aspartate aminotransferase; ALB: albumin; CRP: C-reactive protein; CT: Computed tomography; DBIL: Direct bilirubin; ERCP: Endoscopic retrograde cholangiography; HGB: Hemoglobin; IgG: Immunoglobulin G; IgG4: Immunoglobulin G4 subtype; IgG4-RD: IgG4- related disease; MRI: Magnetic resonance imaging; PET: positron emission tomography; PLT: Platelet; TBIL: Total bilirubin}

\section{Acknowledgements}

We are grateful to Prof. Yin Gen of the Department of General Medicine, West China Hospital of Sichuan University for her contribution to the case. 


\section{Availability of data and materials}

All data used and analysed during this study available from the corresponding author on reasonable request.

\section{Authors' contributions}

DXX wrote the manuscript under the close supervision of FRH. FRH guaranteed the integrity of entire study. DXX prepared the literature search. DXX and FRH designed the study preliminarily and contributed to data acquisition and data analysis. ZJS and LRQ participated in the design and coordination of the study All authors critically revised the manuscript and approved it.

\section{Ethics approval and consent to participate}

This article is a retrospective study and does not contain any studies with human subjects performed by any of the authors. So, the ethical approval was not necessary and West China Hospital of Sichuan University ethics committee can offer exempt ethical statement in support.

\section{Consent for publication}

Written informed consent was obtained from the patient for publication of this case report and any accompanying images. A copy of the written consent is available for review by the editor of this journal.

\section{Competing interests}

The authors declare that they have no competing interests.

\section{Publisher's Note}

Springer Nature remains neutral with regard to jurisdictional claims in published maps and institutional affiliations.

Received: 16 May 2018 Accepted: 29 August 2018

Published online: 04 September 2018

\section{References}

1. Decker L, Crawford AM. Orenzo GL, et al. IgG4-related Hypophysitis: case report and literature review. Cureus. 2016;8:e907.

2. Zhang W, Xue F, Liu MC, et al. 36 cases lgG4 related clinical analysis. [Chinese] Chinese. Journal of Nephrology. 2016;32:253-8.

3. Li YM, Wang H, Fang F, et al. lgG4 related clinical feature analysis. [Chinese] National Medical Journal of China. 2015;95:3281-4.

4. Stone JH, Zen Y, Deshpande V. IgG4-related disease. N Engl J Med. 2012 366:539-51.

5. Woo CG, Yook JH, Kim AY, et al. IgG4-related disease presented as a muralmass in the stomach. J Pathol Transl Med. 2016:50:67-70.

6. Masaki Y, Shimizu H, Nakamura TS, et al. IgG4-related disease: diagnostic methods and therapeutic strategies in Japan. J Clin Exp Hematop. 2014;54: 95-101.

7. Koizumi S, Kamisawa T, Kuruma S, et al. Immunoglobulin G4-related gastrointestinal diseases, are they immunoglobulin G4-related diseases. WJG. 2013;19:5769-74.

8. Umehara H, Okazaki K, Masaki Y, et al. Comprehensive diagnostic criteria for lgG4-related disease (IgG4-RD), 2011. Modern Rheumatology. 2012;22:21-30

9. Umemura T, Zen Y. IgG4-related Hepatopathy. In: Umehara H, Okazaki K, Stone J, Kawa S, Kawano M, editors. IgG4-Related Disease. Tokyo: Springer; 2014. p. 205-9.

10. Hubers LM, Maillette de Buy $L$, Wenniger ME, et al. IgG4-associated cholangitis: a comprehensive review. Clin Rev Allergy Immunol. 2015;48; 198-206.

11. Fong WW, Thumboo J, Azhar R, et al. IgG4-related disease in Singapore: a description of two cases and review of the literature. Int J Rheum Dis. 2013; 16:93-7.

12. Rungsakulkij N, Sornmayura P, Tannaphai P, et al. Isolated IgG4-related sclerosing cholangitis misdiagnosed as malignancy in an area with endemic cholangiocarcinoma: a case report. BMC Surg. 2017;17:17.

13. Cai J, Mou YP, Pan Y, et al. Immunoglobulin G4-associated cholangitis mimicking cholangiocarcinoma treated by laparoscopic choledochectomy with intracorporeal roux-en-Y hepaticojejunostomy. World J Surg Oncol. 2014; 12:363.

14. Euch ME, Hddad S, Mahfoudhi M, et al. A case of type 1 autoimmune pancreatitis (AIP), a form of lgG4-related disease (lgG4-RD). Am J Case Rep. 2017;18:822-5.
15. Miki A, Sakuma Y, Ohzawa H, et al. Immunoglobulin G4-related sclerosing cholangitis mimicking hilar cholangiocarcinoma diagnosed with following bile duct resection: report of a case. Int Surg. 2015;100:480-5.

16. Sivakumaran Y, Le Page PA, Becerril-Martinez G, et al. IgG4-related sclerosing cholangitis: the cholangiocarcinoma mimic. ANZ J Surg. 2014;84:486-7.

17. Rodriguez EA, Williams FK. A mass in the junction of the body and tail of the pancreas with negative lgG4 serology: IgG4-related disease with negative serology. Am J Case Rep. 2015;16:305-9.

18. Kondo T, Uehara T. Immunoglobulin G4-related disease with fibroinflammatory lesions in the pleura, bile ducts and pericardium. CMAJ. 2016;188:972.

19. Yang L, Jin P, Sheng JQ. Immunoglobulin G4-related disease (IgG4-RD) affecting the esophagus, stomach,and liver. Endoscopy. 2015;47:E96-7.

20. Miyajima S, Okano A, Ohana M. Immunoglobulin G4-related hepatic inflammatory pseudotumor invading the abdominal wall. Clin J Gastroenterol. 2017:10:57-62.

21. Li Y, Zhou L, Zhao $X$, et al. The importance of lgG4 screening in patients diagnosed with primary sclerosing cholangitis in the past: a case rediagnosed as IgG4-SC after 10 years. Medicine (Baltimore). 2016;95(50):e5628.

22. Chen $\mathrm{CT}$, Chiang $\mathrm{CL}$, Huang HH. lgG4-related double duct sign mimicking pancreatic head cancer. QJM. 2016:109:207-8.

23. Inoue K, Okubo T, Kato T, et al. IgG4-related stomach muscle lesion with a renal pseudotumor and multiple renal rim-like lesions: A rare manifestation of IgG4-related disease. Mod Rheumatol. 2018;28:188-92.

24. Bulanov D, Arabadzhieva E, Bonev S, et al. A rare case of IgG4-related disease: a gastric mass, associated with regional lymphadenopathy. BMC Surg. 2016:16:37.

25. Takasumi M, Miyata M, Kuroda M, et al. Overlap of IgG4-related disease and primary biliary cirrhosis complicated with autoimmune thrombocytopenia. Internal Med. 2016;55:1387-92.

26. Kim S, Bae H, Choi M, et al. Isolated mass-forming lgG4-related cholangitis as an initial clinical presentation of systemic lgG4-related disease. J Pathol Transl Med. 2016;50:300-5.

27. Matsunaga K, Hayashi R, Otsuka T, et al. A case of IgG4-related disease complicated by duodenal bulbitis with IgG4-positive plasma cell infiltration. Endoscopy. 2014:46:E408-10.

28. Van de Ven AAJM, Seidl M, Drendel V, et al. IgG4-related disease in autoimmune lymphoproliferative syndrome. Clin Immunol. 2017:180:97-9.

\section{Ready to submit your research? Choose BMC and benefit from:}

- fast, convenient online submission

- thorough peer review by experienced researchers in your field

- rapid publication on acceptance

- support for research data, including large and complex data types

- gold Open Access which fosters wider collaboration and increased citations

- maximum visibility for your research: over $100 \mathrm{M}$ website views per year

At BMC, research is always in progress.

Learn more biomedcentral.com/submissions 